\title{
A TEST OF THE REFINEMENT PROCEDURE FOR DETERMINING THE CRYSTALLITE ORIENTATION DISTRIBUTION: POLYETHYLENE TEREPHTHALATE
}

\author{
W. R. KRIGBAUM and ANNA MARIE HARKINS VASEK \\ Gross Chemical Laboratory, Duke University, Durham, North Carolina, U.S.A.
}

(Received October 12, 1971)

\begin{abstract}
A test of the refinement procedure for improving the crystallite orientation distribution function is presented for a fiber texture sample of polyethylene terephthalate. This is a particularly difficult example because the triclinic unit cell offers no simplification due to symmetry, and the pole figures are sharply peaked. The analysis employed 17 observed pole figures and an additional 29 unobserved pole figures reconstructed from the crystallite orientation distribution function. After three cycles of refinement, in which the maximum value of the coefficient was increased from 6 to 16 , the standard deviations, $\sigma_{q}$ and $\sigma_{w}$, of the plane-normal and crystallite orientation distributions were reduced by about a factor of 3 . The refined crystallite orientation distribution function indicates that the $c$-axis tends to align along the fiber axis for this polyethylene terephthalate sample,
\end{abstract}

\section{INTRODUCTION}

In an ideal crystal, the vectors representing the three unit cell translations have quite definite directions. Real single crystals generally have a mosaic structure which has a significant effect on the measured integrated intensities, but the angular divergence is only of the order of minutes of arc. By contrast, one must consider a broad distribution of orientations for a polycrystalline specimen. Operations such as drawing and rolling influence the mechanical properties through changes in the crystallite orientation distribution, hence it is a matter of some practical importance to develop procedures for characterizing this distribution quantitively.

Although the distribution of crystallite orientations may be investigated using a variety of techniques, such as sonic velocity, optical birefringence, infrared dichroism, wide-line NMR, and fluorescence depolarization, the most complete description is furnished by $\mathrm{x}$-ray diffraction. For example, birefringence and infrared dichroism yield only the second moment of the distribution, and the fourth moment can be obtained from NMR and fluorescence depolarization, but only diffraction permits evaluation of the higher moments.

One does not obtain the crystallite orientation distribution directly from the diffraction measurements. The information gained is the angular distribution of the normal to each observed Bragg plane. This may be conveniently represented by the plane-normal distribution, or the pole figure, for each plane. One is then faced with the problem of deducing the crystallite orientation distribution from the diffraction data. Jetter, McHargue and Williams ${ }^{1}$ introduced the concept of the inverse pole figure, and proposed a graphical method for affecting the transformation to this function. Quantitative analytical procedures for deducing the crystallite orientation distribution, or the equivalent inverse pole figure, for samples having fiber symmetry were given by Bunge, ${ }^{2}$ and by Roe and Krigbaum, ${ }^{3}$ and $\mathrm{Roe}^{4}$ has subsequently generalized the latter treatment for application to samples of arbitrary texture. Experimental applications to samples having fiber texture have been presented for aluminum, ${ }^{2}$ polyethylene, ${ }^{5}$ and polyethylene terephthalate, ${ }^{6}$ while Roe's generalized treatment has been successfully applied to biaxially oriented polyethylene samples. $^{7}$

One unfortunate feature of these procedures is that the resolution obtained for the crystallite orientation distribution is limited by the number of pole figures examined. Due to the presence of lattice distortions of the first and second kinds, and the resulting small crystallite sizes, the number of Bragg planes which diffract with appreciable intensity is often quite small. The crystallite orientation distribution is expressed ${ }^{3}$ as a series of poly- 
nomials and, for a given number of measured pole figures, the number of terms in the series which can be retained depends primarily upon the symmetry of the crystal class. Thus, for a sample having fiber texture, the number of pole figures required to evaluate the coefficients $A_{l m}$ and $B_{l m}$ through $l=22$ is: cubic (2), hexagonal (4), trigonal (8), orthorhombic (12), monoclinic (23), and triclinic (45). Moreover, it is often found in practice that the polynomial series must be truncated prior to this theoretical limit because the simultaneous equations become ill-conditioned in the higher coefficients, presumably due to experimental errors in the pole figures. The resolution of the crystallite orientation distribution is, of course, a direct function of the number of terms retained. On the other side, the resolution required in any particular case depends upon the character of the preferred orientation. A small number of terms may suffice if the planenormal distributions are gradual and smooth, whereas a considerably larger number may be required to obtain a satisfactory representation if the plane-normal distributions are sharply peaked.

One of us ${ }^{8}$ has proposed to alleviate this problem through a refinement procedure based upon the analogy between the method used to deduce the crystallite orientation distribution function from pole figure data and that used to solve crystal structures from the measured structure amplitudes. Application of the refinement procedure was illustrated using four pole figures for an isotactic polystyrene sample, which was treated as pseudohexagonal. It was found possible to extend the series beyond the theoretical limit, $l=22$, to $l=36$ by making use of the reconstructed pole figures. The representation achieved, as judged by several criteria, was significantly improved by the refinement. That example concerned a case of high crystal symmetry, and it was recognized that a sample of low crystal symmetry would be a more severe test of the refinement procedure. A test of this type is the subject of the present paper.

\section{REFINEMENT PROCEDURE}

We have selected for this test the pole figure data for one of the four samples of polyethylene terephthalate studied by Krigbaum and Balta. ${ }^{6}$ This is an especially difficult case because it couples the low symmetry of the triclinic class with extremely sharp plane-normal distributions. The particular sample chosen, $S-2$, was prepared by melting and quenching, drawing at the glass transition temperature $\left(70^{\circ} \mathrm{C}\right)$ to an elongation ratio of 3.0, and crystallizing at fixed extension for 45 minutes at $159^{\circ} \mathrm{C}$. The crystallinity as estimated from the measured density was $27.5 \%$. Pole figure data were obtained for 17 reflections. Analysis according to the Roe-Krigbaum procedure could include, as an upper limit, the 45 coefficients $A_{l m}$ and $B_{l m}$ through $l=8$. In actual fact, Krigbaum and Balta ${ }^{6}$ found the best representation to be obtained by retaining only the 28 coefficients through $l=6$. During the course of the refinement procedure, unobserved pole figures were calculated for an additional 29 planes. The pertinent crystallographic data for all 46 planes appear in Table I. The observed planes are numbered 1-17 in column one. Column three lists the $2 \theta$ values, where $\theta$ is the Bragg angle, while columns four and five list the polar and azimuthal angles, $\Theta_{i}$ and $\Phi_{i}$, which locate each reciprocal lattice vector, $\mathbf{r}_{i}$, in the coordinate frame, $X Y Z$, of the unit cell (see Figure 1). These values are based upon the unit cell parameters given for polyethylene terephthalate by Bunn and co-workers, ${ }^{9} a=4.54, b=5.94, c=$ $10.75 \AA, \alpha=98.5^{\circ}, \beta=118.0^{\circ}$ and $\gamma=112.0^{\circ}$.

We begin our analysis by solving the crystallite orientation distribution function, $w(\xi, \phi)$, for the coefficients $A_{l m}$ and $B_{l m}$ through $l_{\max }=6$. We have

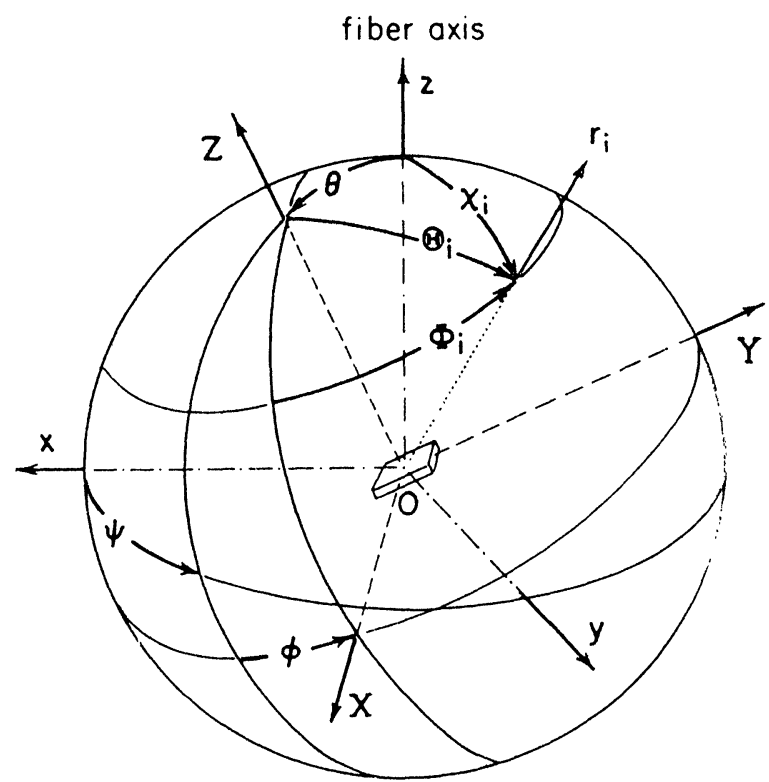

FIGURE 1 Eulerian angles $\theta, \psi$ and $\phi$ (giving orientation of crystallite with respect to sample coordinates $x, y, z)$ and $\Theta_{i}$ and $\Phi_{l}$ (locating reciprocal lattice vector $\mathbf{r}_{l}$ with respect to crystallographic axes $X, Y, Z)$. 
TABLE 1

Crystallographic data for all plane-normals used in the analysis

\begin{tabular}{rrrrr}
\hline$i$ & $h k l$ & $2 \theta$ & \multicolumn{1}{c}{$\Theta_{i}$} & \multicolumn{1}{c}{$\Phi_{i}$} \\
\hline 1 & $0 \overline{1} 1$ & 16.42 & 59.85 & 257.43 \\
2 & 010 & 17.53 & 90.00 & 59.44 \\
3 & $\overline{1} 11$ & 21.31 & 67.24 & 123.86 \\
4 & $\overline{1} 10$ & 22.54 & 90.00 & 138.02 \\
5 & $\overline{1} 12$ & 24.71 & 47.16 & 106.30 \\
6 & 100 & 25.69 & 90.00 & 0.00 \\
7 & $\overline{1} 03$ & 26.44 & 20.02 & 155.79 \\
8 & $1 \overline{1} 1$ & 27.84 & 72.72 & 328.48 \\
9 & 003 & 30.77 & 35.81 & 11.96 \\
10 & $\overline{1} \overline{1} 3$ & 32.44 & 39.75 & 213.62 \\
11 & $0 \overline{2} 1$ & 32.73 & 75.26 & 247.48 \\
12 & $\overline{1} 05$ & 42.66 & 9.70 & 61.00 \\
13 & $0 \overline{2} 4$ & 42.73 & 38.07 & 282.20 \\
14 & $\overline{2} 10$ & 45.91 & 90.00 & 160.43 \\
15 & $\overline{1} 24$ & 47.38 & 44.44 & 231.21 \\
16 & $\overline{2} 05$ & 48.54 & 29.26 & 164.53 \\
17 & $\overline{1} 31$ & 49.04 & 80.05 & 81.60 \\
18 & 112 & 53.91 & 71.56 & 20.80 \\
19 & 120 & 54.83 & 90.00 & 34.75 \\
20 & 023 & 56.93 & 63.17 & 43.85 \\
21 & 021 & 40.89 & 78.15 & 53.04 \\
22 & $\overline{2} 33$ & 57.52 & 63.44 & 98.27 \\
23 & $\overline{2} 31$ & 52.47 & 80.66 & 112.02 \\
24 & $\overline{2} 15$ & 49.64 & 31.33 & 122.08 \\
25 & $\overline{2} 12$ & 39.77 & 65.06 & 150.32 \\
26 & $\overline{2} 11$ & 41.72 & 78.38 & 155.98 \\
27 & 201 & 47.49 & 79.74 & 178.45 \\
28 & $\overline{1} 11$ & 33.34 & 75.52 & 205.71 \\
29 & $\overline{1} \overline{2} 2$ & 46.44 & 68.67 & 221.01 \\
30 & $0 \overline{3} 1$ & 50.93 & 80.40 & 244.59 \\
31 & $1 \overline{3} 0$ & 46.38 & 90.00 & 268.67 \\
32 & $0 \overline{3} 5$ & 59.29 & 43.54 & 273.80 \\
33 & $1 \overline{3} 1$ & 46.13 & 79.45 & 276.22 \\
34 & $2 \overline{3} 1$ & 56.21 & 81.24 & 304.67 \\
35 & $1 \overline{2} 2$ & 38.26 & 64.05 & 305.15 \\
36 & $1 \overline{2} 3$ & 45.22 & 55.97 & 313.74 \\
37 & $2 \overline{2} 1$ & 51.05 & 80.42 & 323.67 \\
38 & 112 & 35.64 & 62.05 & 336.05 \\
39 & $2 \overline{1} 1$ & 52.54 & 80.68 & 343.99 \\
40 & $\overline{2} 32$ & 53.94 & 71.56 & 105.20 \\
41 & $\overline{1} \overline{2} 6$ & 57.31 & 26.20 & 249.24 \\
42 & $\overline{1} 20$ & 31.47 & 90.00 & 104.60 \\
43 & $1 \overline{1} 3$ & 44.72 & 55.56 & 341.61 \\
44 & $\overline{1} 23$ & 43.95 & 54.91 & 74.24 \\
45 & $2 \overline{3} 0$ & 53.25 & 90.00 & 298.55 \\
46 & $1 \overline{1} 0$ & 22.63 & 90.00 & 318.02 \\
& & & & \\
\hline & & & & \\
\hline
\end{tabular}

therefore begun at precisely the terminal point of the analysis presented by Krigbaum and Balta. In order to indicate the extent to which the information content of the observed pole figures is actually utilized, plane-normal distributions, $q_{i}\left(\zeta_{i}\right)$, were recalculated at various values of $\zeta_{i}=\cos \chi_{i}$ for the 17 observed planes through use of the relation

$$
q_{i}\left(\zeta_{i}\right)=\sum_{i=0}^{j} Q_{i}^{i} P_{l}\left(\zeta_{i}\right)
$$

where $P_{l}\left(\zeta_{i}\right)$ are the normalized Legendre polynomials and the series is terminated at $l_{\max }=\lambda$. The coefficients, $Q_{i}^{i}$ are obtained from

$$
Q_{l}^{i}=\int_{-1}^{1} q_{i}\left(\zeta_{i}\right) P_{l}\left(\zeta_{i}\right) d \zeta_{i}
$$

Some typical results are illustrated in Figures 2 and 3 , which show the distributions for the $(003)$ and (111) planes. In each case the circles represent the observed plane-normal distribution, while the full and dashed curves show the series approximations obtained upon truncating at $\lambda=16$ and 6 , respectively. As can be seen in Figure 2, the (003) planenormal distribution is fairly well represented with $\lambda=6$, although its maximum has been considerably broadened. On the other hand, truncation at the same level produces a very poor result for the (111) plane-normal distribution, as shown in

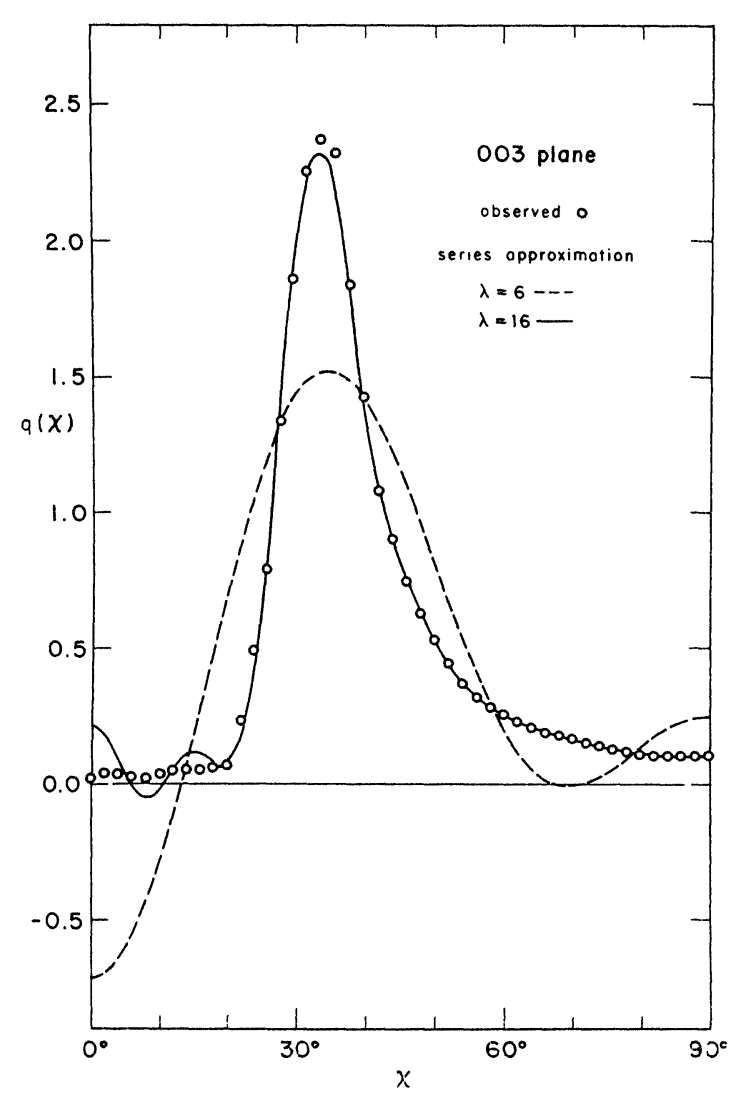

FIGURE 2 Comparison of the observed (003) planenormal distribution (circles) with the polynomial representation obtained using $l_{\max }=\lambda=6$ (dashed curve) and 16 (full curve). 
Figure 3. It should be clearly pointed out that solution of the crystallite orientation distribution function, $w(\xi, \phi)$, with $l_{\max }=6$ involves as input data the representations illustrated by the dashed curves in Figures 2 and 3, not the measured pole figures. Hence, at this limit one is making rather

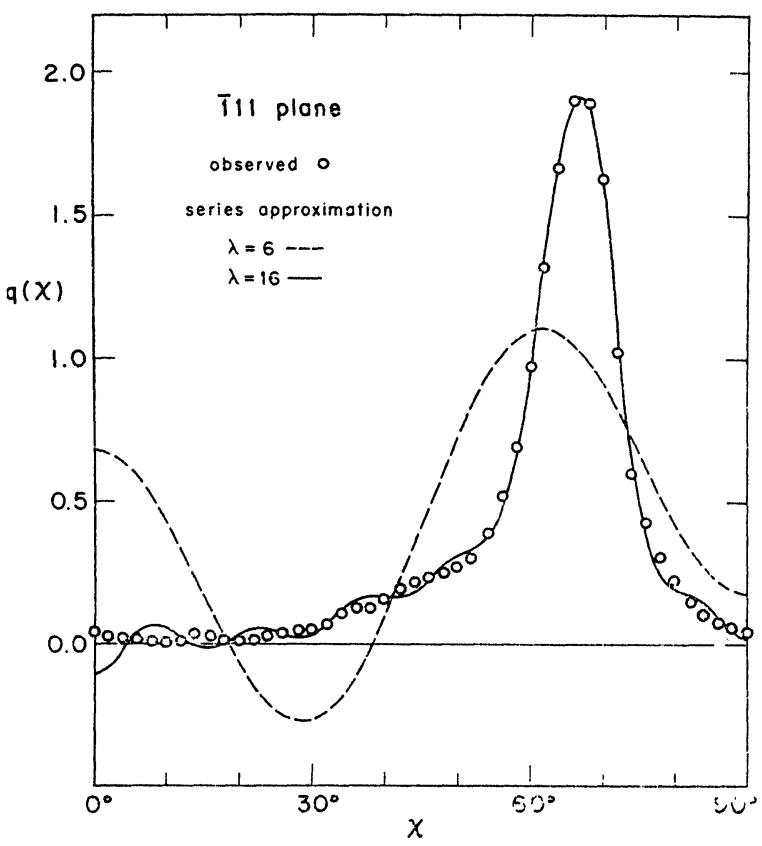

FIGURE 3 Same as Figure 2 for the (111) plane.

poor use of the information content of the observed pole figures. A much better representation of the plane-normal distributions is obtained, of course, with $\lambda=16$.

The plane-normal distributions were next reconstructed for the 20 unobserved planes numbered 20-39 in Table I. The coefficients $Q_{l}^{i}$, appearing in Eq. (1) were calculated from the crystallite orientation distribution through use of the relation

$$
\begin{aligned}
Q_{l}^{i}= & 2 \pi\left(\frac{2}{2 l+1}\right)^{1 / 2}\left\{A_{l 0} P_{l}\left(\cos \Theta_{i}\right)+2 \sum_{m=1}^{l}(-1)^{m}\right. \\
& \left.\times\left[A_{l m} \cos m \Phi_{i}-B_{l m} \sin m \Phi_{i}\right] P_{l}^{m} \cos \Theta_{i}\right\}
\end{aligned}
$$

We then required some indication of the effect of series termination errors upon these reconstructed pole figures. For this purpose the measured pole figures can be compared with those reconstructed using Eqs. (1) and (3), as illustrated for the (003) plane in Figure 4. Using as a guide comparison of the 17 measured pole figures with their series represen- tations when $\lambda=6$, the curves for the 20 unobserved planes were smoothed and sharpened. The peaks were narrowed and moved toward their expected $\chi$ values, and series termination ripples were smoothed out. Figures 5 and 6 illustrate these operations for the (120) and (122) planes. The series approximation terminated at $\lambda=6$ is depicted by the dashed lines, while the full curves indicate the smoothed distributions. We will return to these Figures shortly to discuss the dotted curves.

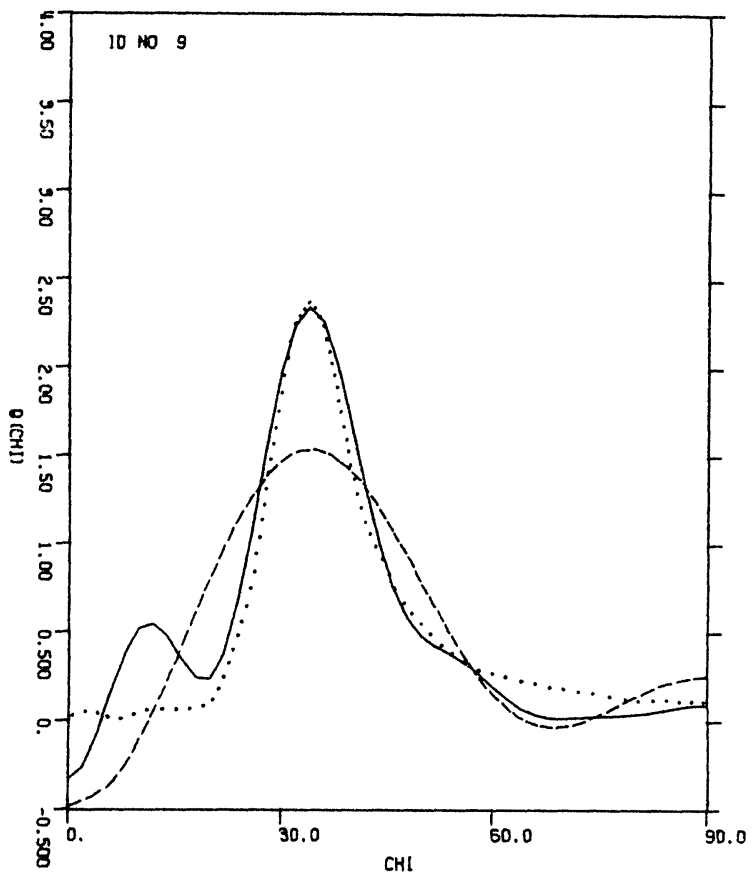

FIGURE 4 Comparison of the observed (003) planenormal distribution with that reconstructed from the crystallite orientation distribution using $\lambda=6$ (dashed curve) and 16 (full curve).

In the first refinement cycle, which utilized the 17 observed and 20 smoothed, unobserved pole figures, the maximum value of $l$ retained was increased to 14 . Hence, this solution for the crystallite orientation distribution function, $w(\xi, \phi)$, makes much more effective use of the measured pole figures. This first refined version of $w(\xi, \phi)$ was employed to recalculate the 20 unobserved planenormal distributions to a better approximation, $\lambda=16$. These were again smoothed, although the changes required in the second smoothing were more modest. These 37 pole figures were used in the second refinement cycle to solve $w(\xi, \phi)$ with $\lambda=16$. At this stage the number of unmeasured 
pole figures which were reconstructed from the crystallite orientation distribution function was increased to 29 , the additional planes appearing in Table I as $18,19,40,41,43-47$. These 29 unmeasured pole figures were reconstructed with $\lambda=18$, smoothed, and combined with the 17 measured pole figures to solve $w(\xi, \phi)$ through $l=18$ in the third cycle. In principle, only 37 of these 46 pole figures are required to solve the crystallite orientation distribution function through $l=18$. Five pole figures $(12,18,30,35$ and 37$)$ which were judged to be poorly behaved, were eliminated, and the crystallite orientation function was again solved through $l=18$.

\section{EVALUATION OF THE REFINEMENT PROCEDURE}

One can see from Figures 2 and 3 that, as $l_{\max }$ is increased during the refinement procedure, there is a noticeable improvement in the extent to which the information contained in the measured pole figures can be utilized in solving for the crystallite orientation distribution function. Correspondingly, when the pole figures for the observed planes are reconstructed from the crystallite orientation distribution function, as illustrated in Figure 4, these resemble the observed pole figures more closely as $\lambda$ is increased. This clearly reflects an improvement in the crystallite distribution function during refinement. We presume that the reconstructed pole figures for the unobserved planes are similarly improved, although in this case we can only observe a closer resemblance to the smoothed profiles as $\lambda$ is increased, as indicated by comparing the dotted and full curves in Figures 5 and 6.

A more quantitative assessment is possible through the standard deviation, $\sigma_{q}$, of the planenormal distribution, defined by

$$
\sigma_{\dot{q}}^{2}=\int_{-1}^{1} q(\zeta) d \zeta-\sum_{l=0}^{\lambda}\left(Q_{l}^{i}\right)^{2}
$$

where the $Q_{l}^{i}$ are calculated from the pole figures using Eq. (2). In order to estimate $\sigma_{q}$ for the unobserved planes, we have taken for $q(\zeta)$ the smoothed profiles from the second cycle $(\lambda=16)$, since these were used as input data for the final cycle. This standard deviation furnishes a measure of series termination effects. If the $Q_{l}^{i}$ are calculated from the crystallite orientation distribution function using Eq. (4), the resulting standard deviation, designated $\sigma_{2}$, reflects errors in the crystallite c

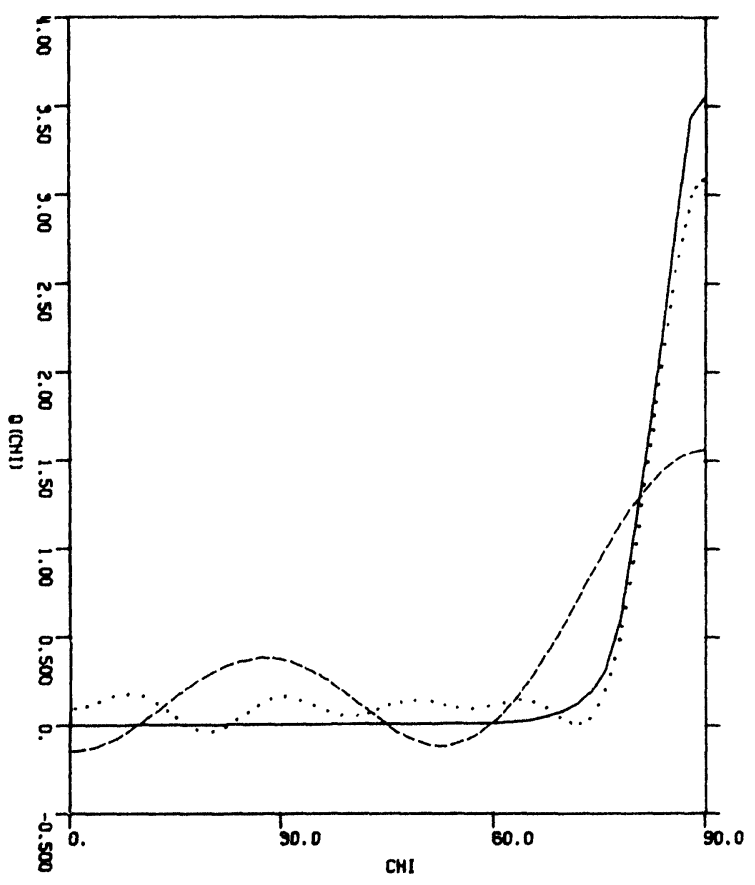

FIGURE 5 Illustration of the smoothing procedure. The dashed curve represents the reconstructed plane-normal distribution for the (120) plane with $\lambda=6$, while the smoothed representation is shown by the full curve. The dotted curve illustrates the reconstructed distribution with $\lambda=16$.

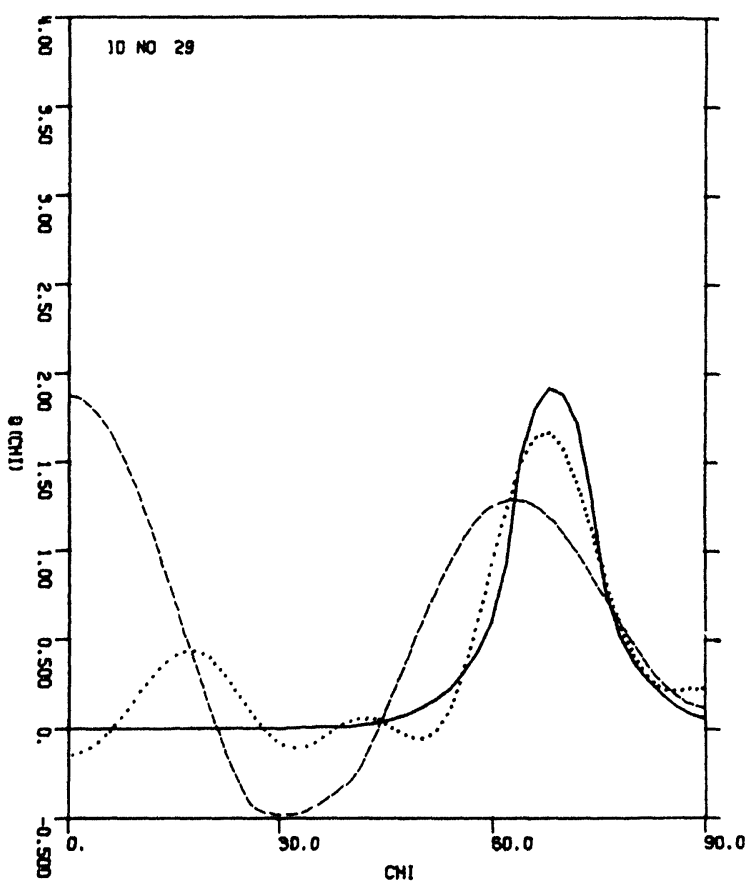

FIGURE 6 Same as Figure 5 for the (122) plane. 


\section{TABLE II}

Standard deviations for the plane-normal distributions

\begin{tabular}{|c|c|c|c|c|c|}
\hline \multirow{2}{*}{ Plane no. } & \multicolumn{2}{|c|}{ Values of $\sigma_{\boldsymbol{q}}$} & \multicolumn{3}{|c|}{ Values of $\sigma_{2}$} \\
\hline & $\lambda=6$ & $\lambda=18$ & $\lambda=6$ & $\lambda=18^{\mathrm{a}}$ & $\lambda=18^{b}$ \\
\hline 1 & 0.385 & 0.153 & 0.579 & 0.427 & 0.384 \\
\hline 2 & 0.283 & 0.045 & 0.505 & 0.229 & 0.239 \\
\hline 3 & 0.372 & 0.154 & 0.517 & 0.414 & 0.391 \\
\hline 4 & 0.495 & 0.212 & 0.737 & 0.415 & 0.313 \\
\hline 5 & 0.455 & 0.191 & 0.676 & 0.378 & 0.357 \\
\hline 6 & 0.376 & 0.088 & 0.634 & 0.278 & 0.367 \\
\hline 7 & 0.577 & 0.231 & 0.689 & 0.682 & 0.682 \\
\hline 8 & 0.577 & 0.162 & 0.717 & 0.353 & 0.393 \\
\hline 9 & 0.405 & 0.144 & 0.416 & 0.395 & 0.393 \\
\hline 10 & 0.131 & 0.032 & 0.254 & 0.537 & 0.551 \\
\hline 11 & 0.189 & 0.059 & 0.136 & 0.391 & 0.387 \\
\hline 12 & 0.550 & 0.252 & 1.723 & 2.332 & - \\
\hline 13 & 0.178 & 0.085 & 0.278 & 0.709 & 0.742 \\
\hline 14 & 1.193 & 0.239 & 2.502 & 2.429 & 2.451 \\
\hline 15 & 0.243 & 0.040 & 0.309 & 0.429 & 0.455 \\
\hline 16 & 0.323 & 0.162 & 0.381 & 0.833 & 0.846 \\
\hline 17 & 0.383 & 0.213 & 0.767 & 0.628 & 0.643 \\
\hline 18 & & 0.204 & & 0.100 & - \\
\hline 19 & & 0.144 & & 0.698 & 0.747 \\
\hline 20 & & 0.171 & & 0.442 & 0.409 \\
\hline 21 & & 0.126 & & 0.391 & 0.370 \\
\hline 22 & & 0.207 & & 0.458 & 0.464 \\
\hline 23 & & 0.363 & & 0.501 & 0.483 \\
\hline 24 & & 0.079 & & 0.523 & 0.526 \\
\hline 25 & & 0.056 & & 0.453 & 0.431 \\
\hline 26 & & 0.107 & & 0.640 & 0.620 \\
\hline 27 & & 0.263 & & 0.358 & 0.376 \\
\hline 28 & & 0.152 & & 0.419 & 0.443 \\
\hline 29 & & 0.162 & & 0.189 & 0.251 \\
\hline 30 & & 0.138 & & 0.137 & - \\
\hline 31 & & 0.041 & & 0.280 & 0.379 \\
\hline 32 & & 0.039 & & 0.301 & 0.284 \\
\hline 33 & & 0.164 & & 0.103 & 0.099 \\
\hline 34 & & 0.253 & & 0.189 & 0.231 \\
\hline 35 & & 0.225 & & 0.304 & - \\
\hline 36 & & 0.156 & & 0.394 & 0.332 \\
\hline 37 & & 0.373 & & 0.498 & - \\
\hline 38 & & 0.063 & & 0.251 & $\overline{0.192}$ \\
\hline 39 & & 0.141 & & 0.228 & 0.284 \\
\hline 40 & & 0.234 & & 0.429 & 0.455 \\
\hline 41 & & 0.051 & & 0.304 & 0.084 \\
\hline 42 & & 0.134 & & 0.664 & $\begin{array}{l}0.688 \\
0.688\end{array}$ \\
\hline 43 & & 0.154 & & 0.114 & $\begin{array}{l}0.000 \\
0.128\end{array}$ \\
\hline 44 & & 0.178 & & 0.587 & 0.524 \\
\hline 45 & & 0.132 & & 0.404 & 0.472 \\
\hline 46 & & 0.129 & & 0.616 & 0.673 \\
\hline Av. & 0.419 & 0.155 & 0.696 & 0.496 & 0.476 \\
\hline
\end{tabular}

a Calculated in third cycle using 46 planes.

${ }^{b}$ Calculated in third cycle using 41 planes. orientation distribution function as well as series termination effects. Values for $\sigma_{q}$ and $\sigma_{2}$ appear in Table II. Comparison of the average values shows that both $\sigma_{q}$ and $\sigma_{2}$ are significantly reduced by the refinement process. The two columns labeled $\lambda=18$ indicate that the crystallite orientation distribution was not significantly improved by deleting the five planes which were judged to be poorly behaved. This means either that the selection of poorly behaved planes was unsuccessful, or that there was sufficient redundancy in the collection of 47 planes so that errors in the individual pole figures could be minimized by the least-squares procedure in solving for $\lambda=18$.

The crystallite orientation distribution is expressed in terms of the angles $\theta$ and $\phi$ shown in Figure 1. For this triclinic unit cell, the orthogonal crystallographic axes $X, Y, Z$ are taken as $a^{*}, b^{*}$ and $c$. According to the Roe-Krigbaum treatment, ${ }^{3}$ the crystallite orientation distribution function may be expressed in series form:

$$
\begin{aligned}
w(\xi, \phi)= & \sum_{l=0} A_{l 0} P_{l}^{0}(\xi) \div 2 \sum_{l=2}^{\infty} \sum_{m=1}^{l} \\
& {\left[A_{l m} \cos m \phi+B_{l m} \sin m \phi\right] P_{l}^{m}(\xi) }
\end{aligned}
$$

where the $P_{l}^{m}(\xi)$ are spherical harmonics in the variable $\xi=\cos \theta$, and $l$ takes only even values. If the series is truncated at $l_{\max }=\lambda$, the standard deviation, $\sigma_{w}$, of this finite series is

$$
\sigma_{w}^{2}=2 \pi \sum_{l=\lambda+2}^{\infty}\left[A_{10}^{2}+2 \sum_{m=1}^{l}\left(A_{l m}^{2}+B_{l m}^{2}\right)\right]
$$

Since the coefficients having $l>\lambda$ are unknown, Roe and Krigbaum have proposed the following approximation :

$$
\left\langle Q_{l}^{2}\right\rangle \cong 2 \pi^{2}\left(\frac{2}{2_{l}+1}\right)\left[A_{l 0}^{2}+2 \sum_{m=1}^{l}\left(A_{l m}^{2}+B_{l m}^{2}\right)\right]
$$

where $\left\langle Q_{l}^{2}\right\rangle$ represents an average over all observed planes. If this relation can be shown to be a satisfactory approximation, $\sigma_{w}$ may be estimated from

$$
\sigma_{w}^{2} \cong \frac{1}{\pi} \sum_{l=\lambda+2}^{\infty}\left(\frac{2 l+1}{2}\right)\left\langle Q_{l}^{2}\right\rangle
$$

A test of the approximate equality proposed by Roe and Krigbaum is shown in Figure 7, where $\ln \left\langle Q_{l}^{2}\right\rangle$ and the natural logarithm of the righthand side of Eq. (7) are separetely plotted as a function of $l^{2}$. There appears to be a tendency for the circles to fall on a curve having upward curvature. Hence, if we use the approximation 
$\left\langle Q_{l}^{2}\right\rangle=a e^{-b l^{2}}$, and replace the sum in Eq. (8) by an integral, we will obtain a lower bound for $\sigma_{w}$. From the intercept and slope of the line drawn in Figure $7, a=0.246$ and $b=9.3 \times 10^{-3}$, which gives for $\lambda=6,16$ and 18 the $\sigma_{w}$ values $1.56,0.51$ and 0.32 , respectively. On the other hand, we can

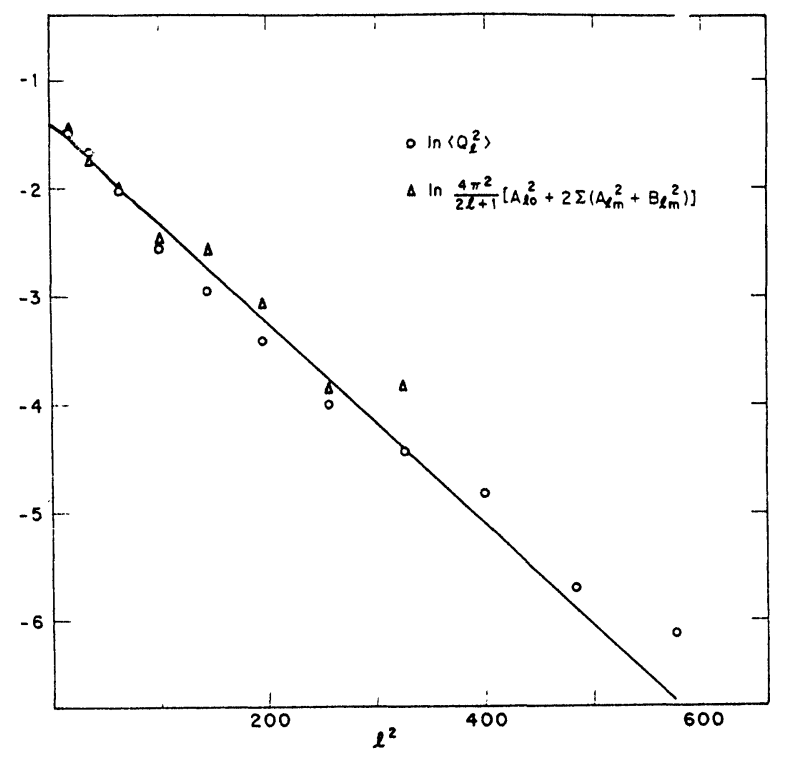

FIGURE 7 A test of Eq. (7).

obtain an upper bound to $\sigma_{w}$ by fitting only the points for large $l$ to $\left\langle Q_{l}^{2}\right\rangle=A e^{-B l}$. This plot (not shown) is concave from below. From the tangent at $l=22, A=2.05$ and $B=0.59$, which furnishes as upper bounds for $\lambda=6,16$ and 18 the $\sigma_{w}$ values $1.63,0.59$ and 0.43 . Krigbaum and Balta ${ }^{6}$ used the first of these procedures; however, they gave an incorrect value for the intercept of their line. If this error is corrected, one obtains from their plot for $l^{2} \leqq 256$ the values $a=0.237$ and $b=1.2 \times 10^{-2}$, which gives a somewhat more optimistic estimate, $\sigma_{w}=1.30$, for $\lambda=6$. We conclude that the refinement procedure has reduced $\sigma_{w}$ by a factor of between three and four, which is a significant improvement.

Finally, we examine how the crystallite orientation distribution function varies during the refinement procedure. The function $G(\xi, \phi)=$ $4 \pi w(\xi, \phi)$ has some advantage for graphical presentation, since a unit value of $G(\xi, \phi)$ indicates that as many crystallites have that particular orientation as would be found in a perfectly random sample. In Figure 8 are compared the $G(\xi, \phi)$ function, calculated with $\lambda=6$ using the
Roe-Krigbaum procedure, and that obtained from the refinement procedure using 46 pole figures. In the latter case $\lambda=16$, which we believe offers the best representation, since maxima which we judge to be spurious begin to appear when $\lambda \geqq 18$. As expected, the $G(\xi, \phi)$ function is considerably sharpened by the refinement procedure, the global maximum increasing from 17.1 at $\lambda=6$ to 53.8 when $\lambda=16$. Of more significance are changes in the details of the $G(\xi, \phi)$ function. We suspect that the contours marked 1, which correspond to $G(\xi, \phi)=2$, are of dubious significance. If these are deleted, the $G(\xi, \phi)$ functions for $\lambda=6$ and 16 are in qualitative agreement. However, a more detailed examination reveals that the maximum moves from $\theta=6^{\circ}$ when $\lambda=6$ toward $\theta=0^{\circ}$ as $\lambda$ isincreased, and the highest (inner) contours begin to resemble circles about the point $\theta=0^{\circ}$. We therefore believe that for fiber texture samples of polyethylene terephthalate the $c$-axis tends to align preferentially along the fiber axis. Heffelfinger and Schmidt ${ }^{10}$ also state that the $c$-axis of polyethylene terephthalate tends to align along the fiber axis, although we are unable to compare their results with ours in any more detailed manner. Daubeny, Bunn and Brown ${ }^{9}$ indicated in their study that the $c$-axis is inclined by approximately $5^{\circ}$ to the fiber axis, and Krigbaum and Balta ${ }^{6}$ reached a similar conclusion from their earlier analysis of the data utilized here.

\section{CONCLUSION}

This example indicates that the refinement procedure can be successful, even in the difficult case presented by a material having triclinic symmetry and sharply peaked pole figures. The improvement is reflected in the fact that the reconstructed pole figures become more faithful representations of those observed, and through the reductions of $\sigma_{q}$ and $\sigma_{w}$. An improvement is expected since the information contained in the observed pole figures can be more effectively utilized as higher terms are added to the series during refinement. Although the refinement procedure permits one to exceed the theoretical limit established by the Roe-Krigbaum treatment, which is $l=8$ in the present case, there does appear to be a second limit beyond which the higher coefficients become ill-conditioned. This behaviour probably arises from mutual inconsistencies among the observed pole figures. In this example the best representation of the crystallite orientation 


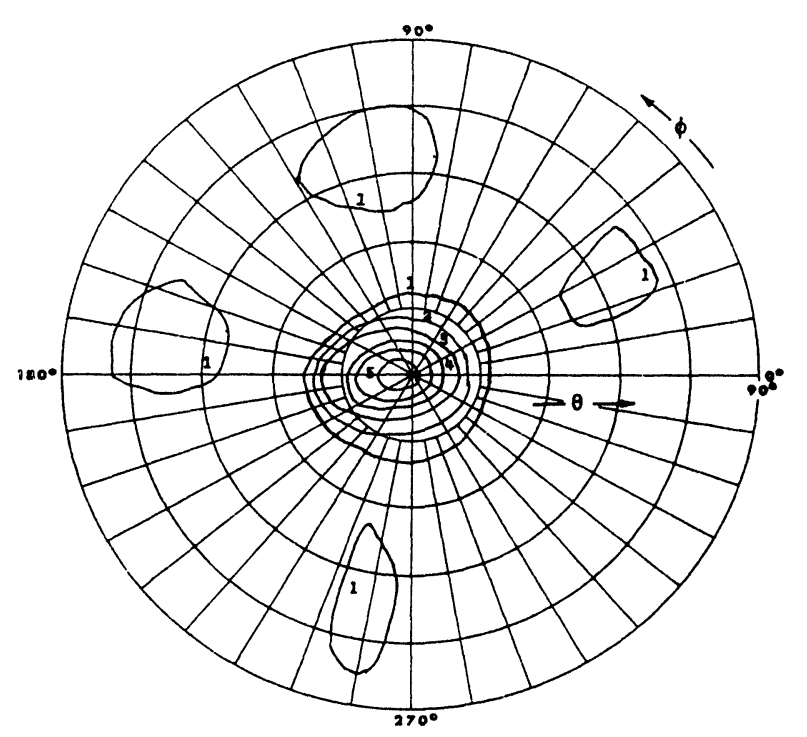

$$
\lambda=6
$$

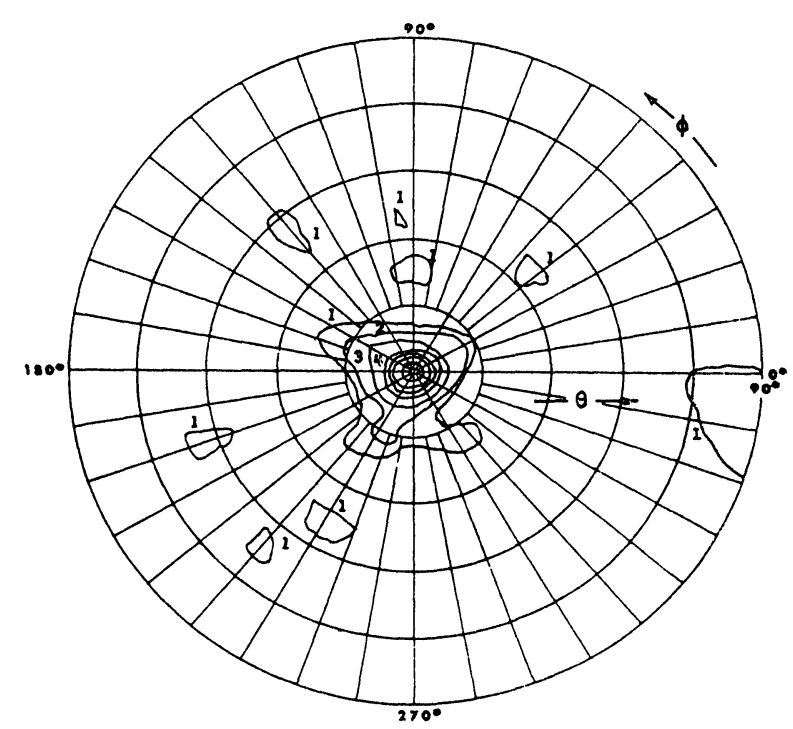

$\lambda=16$

FIGURE 8 Contour maps of $G(\xi, \phi)$ in polar stereographic projection. For $\lambda=6$ contours $1-5$ correspond to probability densities $2,6,10,14$ and 16 , while for $\lambda=16$ contours $1-7$ correspond to probability densities $2,5,11$, $30,40,45$ and 50 .

distribution was obtained with $\lambda=16$. Thus, although the theoretical limit can be exceeded, the refinement procedure does not fully overcome the disadvantage arising from low crystal symmetry. This can be seen by comparison of the present results with those obtained by applying the refinement procedure to the more favourable case of isotactic polystyrene. In the latter case, which was treated as pseudohexagonal, it was possible to extend the series to $\lambda=36$ using only four observed pole figures, and to obtain much smaller standard deviations, $\sigma_{q}=0.037$ and $\sigma_{w}=0.013$.

\section{ACKNOWLEDGEMENT}

This work was supported in part by National Science Foundation grant GP-17873.

\section{REFERENCES}

1. L. T. Jetter, C. J. McHargue and R. O. Williams, J. Appl. Phys. 27, 363 (1956).

2. H. J. Bunge, Monatsber. Deut. Akad. Wiss. Berlin 1, 27, 400 (1959); 3, 97 (1961).

3. R.-J. Roe and W. R. Krigbaum, J. Chem. Phys. 40, 2608 (1964).

4. R.-J. Roe, J. Appl. Phys. 36, 2024 (1965).

5. W. R. Krigbaum and R.-J. Roe, J. Chem. Phys. 41, 737 (1964).

6. W. R. Krigbaum and Y. I. Balta, J. Phys. Chem. 71, 1770 (1967).

7. W. R. Krigbaum, T. Adachi and J. V. Dawkins, J. Chem. Phys. 49, 1532 (1968).

8. W. R. Krigbaum, J. Phys. Chem. 74, 1108 (1970).

9. R. de P. Daubeny, C. W. Bunn and J. C. Brown, Proc. Roy. Soc. (London) A226, 531 (1954).

10. C. J. Heffelfinger and P. G. Schmidt, J. Appl. Poly. Sci. 9, 2661 (1965). 